\title{
Modified structure of graphene oxide by investigation of structure evolution
}

\author{
A NEKAHI, S P H MARASHI* and D HAGHSHENAS FATMESARI \\ Department of Mining and Metallurgical Engineering, Amirkabir University of Technology, Tehran, Iran
}

MS received 26 April 2015; accepted 13 July 2015

\begin{abstract}
The structure of graphite oxide and graphene oxide (GO) has been studied previously using various analyses and computer simulations. Although some oxygen functional groups (OFGs) are accepted as the main functionalities in GO, the structure of GO has remained elusive. In this regard, GO was produced using the modified Hummers method and was investigated using X-ray diffraction pattern, Fourier transform infrared analysis and Boehm titration method. Based on the obtained results, a modified model was proposed for GO based on the model of Lerf-Klinowski. OFGs include highly carboxyl groups and phenols with few epoxides, lactones and ketones agglomerated in some regions due to hydrogen bonding between functional groups. Trapped water molecules were shown between the GO sheets which strongly affected the distribution of OFGs and their aggregation by hydrogen bonding.
\end{abstract}

Keywords. Graphene oxide; oxygen functional groups; structure investigation; modified Hummers method.

\section{Introduction}

Graphene oxide (GO) is a new material with unique physical and chemical properties such as large theoretical specific surface area, which makes it attractive for different applications ${ }^{1}$ including optoelectronics ${ }^{2-4}$ and composite materials. ${ }^{5,6}$ Determining the structure of GO is essential for a better understanding of its fundamental properties. The specific surface area of graphene materials and specially the surface oxygen functional groups (OFGs) determine their applications. Because of these properties, graphene materials have different characteristics; from insulating for GO to completely conductive for pristine graphene.

Various structural models were proposed for GO with respect to the presence of OFGs (figure 1). These structures were proposed based on the results obtained using solidstate nuclear magnetic resonance (NMR), X-ray photoelectron spectroscopy (XPS), Fourier transform infrared (FTIR) analysis, Raman spectra, etc. ${ }^{7-19}$ or by computer simulation mostly by density functional theory (DFT). ${ }^{20-24}$ GO structure is stacking layers of carbon hexagonal planes like graphite oxide with two distinct differences: (i) GO is a monolayer or few-layer carbon hexagonal lattice; whereas, the number of stacked layers in graphite oxide is much more and interlayer distance is so low about $3.3 \AA$, (ii) the content of OFGs in GO is higher than in graphite oxide due to the compaction of graphite structure. ${ }^{25}$ Epoxy groups were the first OFGs spread across the basal flat planes of graphite. ${ }^{7}$ After that, other OFGs including hydroxyl groups,${ }^{8}$ carbonyl groups, ${ }^{9,26}$ carboxyl groups, ${ }^{10}$ ketones ${ }^{17}$ and lactones ${ }^{27}$ were

*Author for correspondence (pmarashi@aut.ac.ir) added to the wrinkled ${ }^{8,17}$ or flat $^{7}$ carbon sheets of graphite oxide according to the results of FTIR, NMR and XPS analysis. Regarding distribution of OFGs on the carbon lattice, there were two opposite ideas: aggregation of the groups were proposed in GO structure ${ }^{18}$ and in opposite, it was mentioned that OFGs were placed completely far from each other to minimize the repulsion between them. ${ }^{16}$ However, the content of OFGs and their placement on the basal carbon lattice is strongly affected by the size of pristine graphite and hence, produced GO sheets. ${ }^{19}$ Some bindings were proposed between different $\mathrm{OFGs}^{11}$ as well as strong hydrogen bonding with water molecules, trapped in the space between the sheets, ${ }^{9}$ or with carbon atoms of the basal planes. ${ }^{9}$

To understand the exact structure of GO, some important works were performed by Klinowski and co-workers. ${ }^{12-15}$ They first proposed graphite oxide structure with $\mathrm{C}=\mathrm{C}, \mathrm{C}-$ $\mathrm{OH}$ and $\mathrm{C}-\mathrm{O}-\mathrm{C}$ bindings. ${ }^{12}$ Interaction of water molecules with structure ${ }^{13}$ and hydrogen bonding between OFGs ${ }^{14}$ were also considered. In addition, $\mathrm{COOH}$ groups were added to the carbon flat basal plane which is slightly distorted in the place of hydroxyl groups. ${ }^{15}$

According to the literature, the attempt of this paper is to propose a new structure for GO. Even though interesting works were performed by Klinowski's group,,${ }^{12-15}$ there were some points which were not mentioned in their works and were suggested by the authors in this work. In this regard, the oxidation process of different graphite flakes and the oxidized structure were investigated during the GO formation process by a modified Hummers method. Moreover, different analyses were carried out on produced GO to determine the content and the kinds of functional groups in the structure of GO. Finally, based on the results and the models by Lerf-Klinowski, ${ }^{14}$ a modified structure is proposed for GO. 


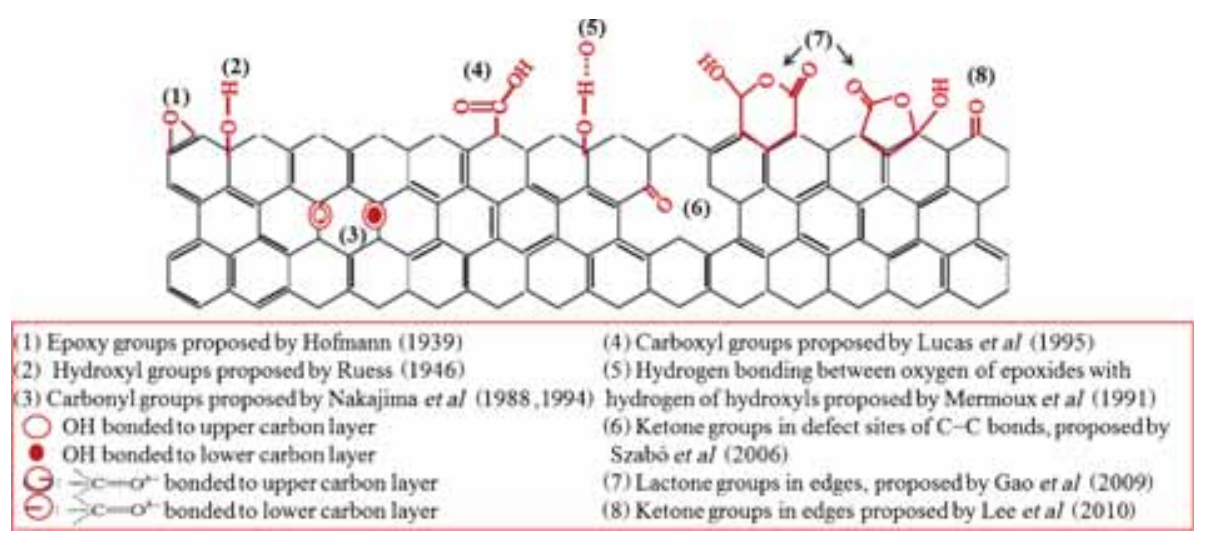

Figure 1. Schematic representation of different oxygen functionalities in graphite oxide and graphene oxide (just for showing different types of functionalities, despite their distribution on carbon plane).

\section{Experimental}

Graphene oxide was prepared using a modified Hummers method from natural graphite flakes $\left(\mathrm{G}_{1}\right.$ : Japan, $\mathrm{G}_{2}$ : Asbury Graphite Mills, US, $\mathrm{G}_{3}$ : EX 9550-200N, Beijing Invention Biology Engineering \& New Material Co., China) in a roundbottom flask according to previous literature. ${ }^{28-30}$ Graphite named $G_{1}$ was pre-treated with acids before expansion. These flakes had the average size of $500 \mu \mathrm{m}$ (figure 2a). Sulphuric acid $\left(\mathrm{H}_{2} \mathrm{SO}_{4}, 95 \%\right.$, Dr. Mojallali Chemical Laboratory, Iran) and fuming nitric acid $\left(\mathrm{HNO}_{3}, 65 \%\right.$, Dr. Mojallali Chemical Laboratory, Iran) were poured slowly and stirred for $24 \mathrm{~h}$. After that, deionized (DI) water was poured into the mixture and then the mixture was filtered and washed using DI water and dried in air to obtain graphite-intercalation compounds (GIC) (figure 2b). $\mathrm{GIC}_{1}$ of $\mathrm{G}_{1}, \mathrm{G}_{2}$ and $\mathrm{G}_{3}$ were thermally expanded separately at $1000^{\circ} \mathrm{C}$ for $10 \mathrm{~s}$. The modified Hummers method for all types of graphite was performed in the same way. One gram of expanded graphite and $100 \mathrm{ml}$ of sulphuric acid were mixed in a three-necked flask and stirred slowly. Nine grams of $\mathrm{KMnO}_{4}$ (Dr. Mojallali Chemical Laboratory, Iran) was added to the mixture for extreme oxidation in a slow rate to avoid temperature raise due to the exothermic reaction. The mixture was stirred for $24 \mathrm{~h}$. Briefly, $200 \mathrm{ml}$ of DI water and $35 \mathrm{ml}$ of $\mathrm{H}_{2} \mathrm{O}_{2}$ (35\%, Dr. Mojallali Chemical Laboratory) were poured sequentially into the mixture; each time it was stirred for $30 \mathrm{~min}$. $\mathrm{H}_{2} \mathrm{O}_{2}$ was added to stop oxidation reaction and to reduce excess $\mathrm{KMnO}_{4}$. The produced suspension was washed by repeated centrifugation $(4000 \mathrm{rpm})$ with a $\mathrm{HCl}(37 \%$, Dr. Mojallali Chemical Laboratory, Iran) solution ( $9: 1$ water : $\mathrm{HCl}$ by volume) and then with DI water until $\mathrm{pH} 7$ was reached. The final suspension was subjected to 5 min sonication for more exfoliation and was centrifuged again. Extreme oxidation with strong acidic media with excess potassium permanganate results in the formation of more OFGs without severe sonication. The sonication inevitably results in severe breakage of graphene sheets into small pieces or causes small holes in the resulting GO nanosheets. ${ }^{31}$
For understanding the structural changes during oxidation, different samples were prepared from $\mathrm{G}_{1}$ : the first graphite flakes $\left(G_{1}\right)$, expanded intercalated graphite $\left(E_{I} G_{1}\right)$, sample before centrifuge (or acid-treated graphite: $\mathrm{ATG}_{1}$ ), and final $\mathrm{GO}\left(\mathrm{GO}_{1}\right)$. Scanning electron microscope (SEM) was used for understanding changes of morphology from graphite to $\mathrm{GO}_{1}$. Increasing interlayer distance was investigated by Xray diffraction (XRD) patterns. FTIR analysis was performed for determining the presence of different functional groups. The same analysis was performed for other GOs from $\mathrm{G}_{2}$ (as $\mathrm{GO}_{2}$ ) and $\mathrm{G}_{3}\left(\right.$ as $\left.\mathrm{GO}_{3}\right)$.

The content of phenol, carboxyl and lactone groups was determined using the Boehm titration method. ${ }^{32} \mathrm{GO}_{1}$ was immersed into different base solutions of $0.1 \mathrm{M} \mathrm{NaOH}, 0.1 \mathrm{M}$ $\mathrm{NaHCO}_{3}$ and $0.05 \mathrm{M} \mathrm{Na}_{2} \mathrm{CO}_{3}$. NaOH neutralizes carboxyl, lactone and phenolic groups, $\mathrm{Na}_{2} \mathrm{CO}_{3}$ neutralizes carboxyl and lactone and $\mathrm{NaHCO}_{3}$ neutralize only carboxyl groups. The base mixtures containing GO were shaken for $24 \mathrm{~h}$ and were titrated with $\mathrm{HCl}(0.1 \mathrm{M})$ to endpoint of the neutral $\mathrm{pH}$.

According to the results, a modified structure was proposed for GO.

\section{Results and discussion}

\subsection{GO production}

Graphite flakes of $\mathrm{G}_{1}$ (figure 1a) were used in the Hummers method for GO production with a pretreatment process. The flakes were reacted with strong acid solution of $\mathrm{H}_{2} \mathrm{SO}_{4}$ and $\mathrm{HNO}_{3}$. According to these reactions, acid molecules $\left(\mathrm{H}_{2} \mathrm{SO}_{4}\right.$ and $\left.\mathrm{HNO}_{3}\right)$ and their ions $\left(\mathrm{HSO}_{4}^{-}, \mathrm{SO}_{4}^{2-}\right.$ and $\mathrm{NO}_{3}^{-}$) can be intercalated in the space between the layers of hexagonal carbon structure of graphite, as schematically represented in figure 3. It should be mentioned that these interlayer species could be drawn after washing with water. Based on XRD results (figure 4; $\mathrm{EIG}_{1}$ ) interlayer distance of graphitic layers was not changed considerably.

After intercalation, $\mathrm{GIC}_{1}$ and $\mathrm{G}_{2}$ and $\mathrm{G}_{3}$ were expanded. The expansion results in the volume increment of graphite 

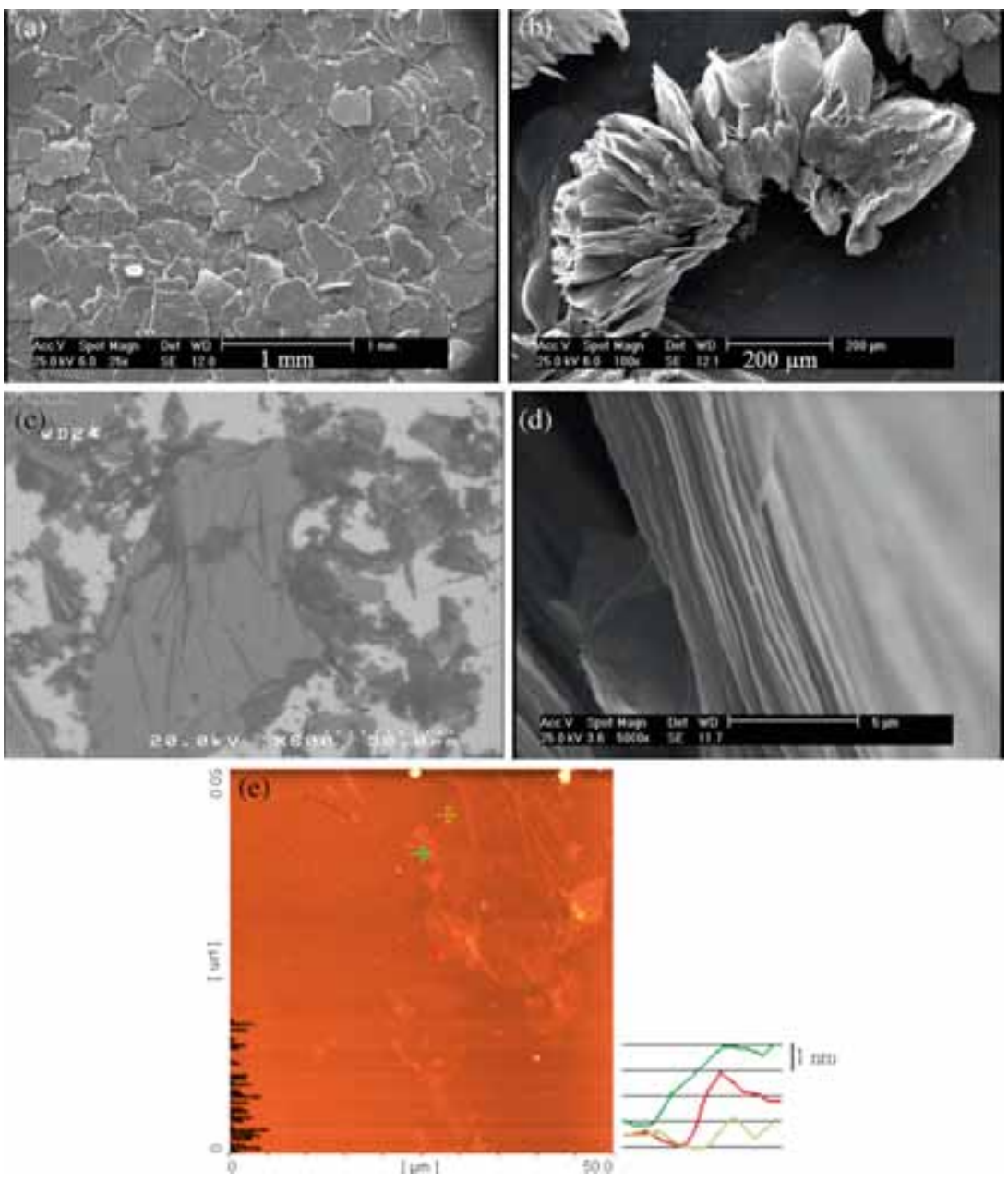

Figure 2. SEM micrographs of (a) graphite flakes, $\mathrm{G}_{1}$; (b) expanded graphite of $\mathrm{G}_{1}$; (c) GO sheets; and (d) layer stacking of GO in film formation. (e) AFM result of graphene oxide showing morphology and thickness of GO sheet.

flakes and producing worm-like structure with big internal pores (figure $2 \mathrm{~b}$ ). Internal pores increased the exposure of graphitic layers to acid during oxidation process. Oxidation of expanded graphite was performed using a mixture of potassium permanganate and sulphuric acid. Simultaneous presence of permanganate and sulphuric acid has an important effect on the extreme oxidation of graphitic structure. $\mathrm{Mn}_{2} \mathrm{O}_{7}$ is the predominant oxidation agent. High amount of permanganate, in excess of the stoichiometric value, results in more extreme oxidation of graphite and therefore, helps in the better separation of graphitic layers from each other.

In following, DI water was added to the mixture. Oxygen is produced from the reaction of added water with potassium permanganate which results to the effervescence and react with graphite flakes. At last, hydrogen peroxide was added to stop the reaction and to dissolve the permanganate salts as colourless sulphates. XRD pattern of sample prepared from this stage is shown in figure $4, \mathrm{ATG}_{1}$. This pattern shows fading of graphite structure. As $\mathrm{ATG}_{1}$ was gel-like, no obvious peak was observed in XRD pattern.
Following the oxidation process, graphite oxide sheets were centrifuged to be separated from each other. In this stage, if the electrostatic force between GO sheets was greater than or equal to the sum of gravity and Van der Waals forces, GO suspension in water is stabilized forever. Owing to the higher oxidation level of GO, electrostatic force is high enough and hence, GO suspension is highly stabilized for a long time. ${ }^{23,33}$ The morphology of GO and stacking of GO layers are shown in figure $2 \mathrm{c}$ and d, respectively. Figure 1e shows atomic force microscopic (AFM) image of a GO sheet with wrinkles. AFM line scans show that the thickness of the GO sheet was $\approx 1 \mathrm{~nm}$, which is due to the presence of oxygen functional groups on the surface. ${ }^{29}$

\subsection{Structure of $G O$}

In previous research works, according to the results of some analysis of GO, some different structures were proposed which are summarized in the introduction section and in figure 1. In this work, FTIR spectroscopy was used to reveal different bindings in GO (figure 5). It should be noted that 
FTIR spectra is sensitive to the synthesis method and its condition and the content of functional groups is not the same for different GOs. FTIR analysis of the specimen before centrifuge is shown in figure 5. This specimen also has OFGs

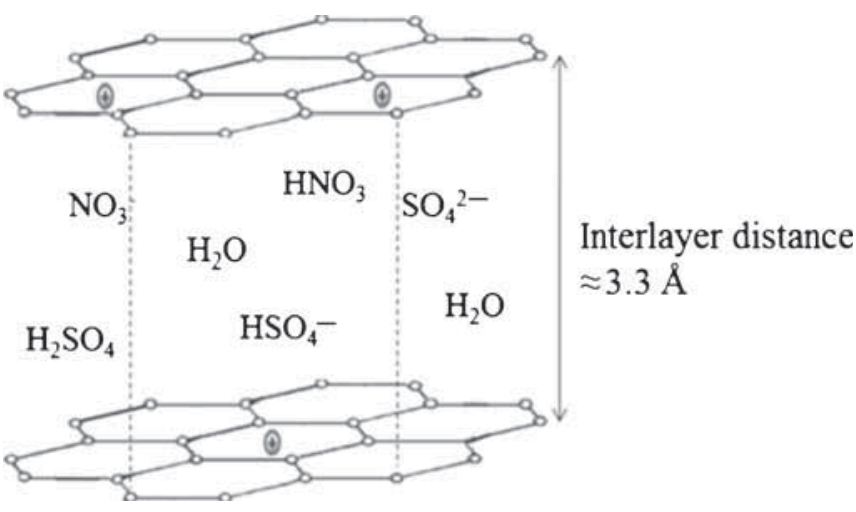

Figure 3. Schematic structure of graphite intercalated with $\mathrm{HNO}_{3}$ and $\mathrm{H}_{2} \mathrm{SO}_{4}$.

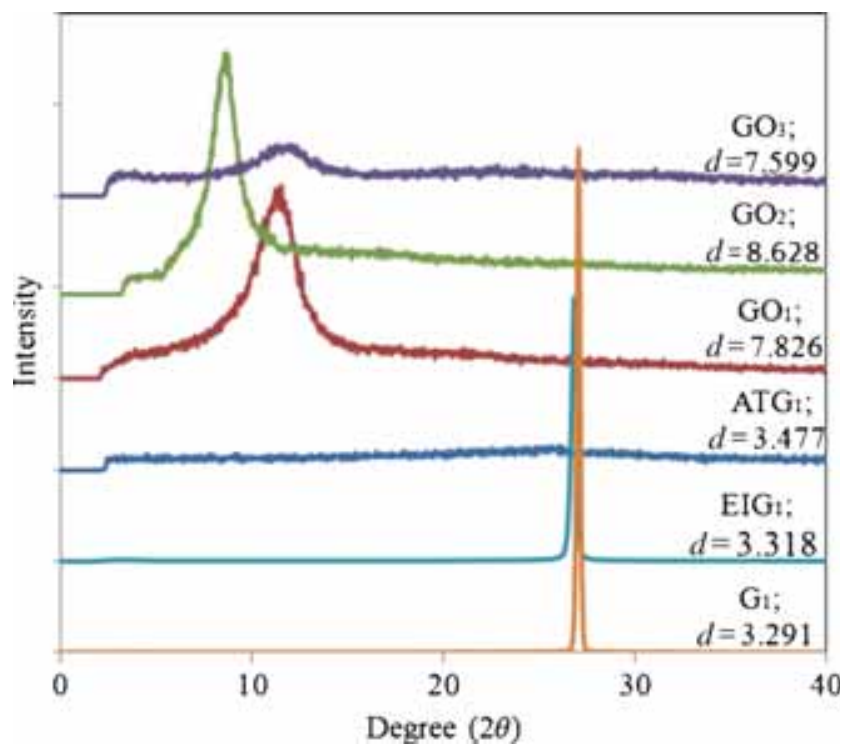

Figure 4. XRD spectra of $\mathrm{G}_{1}, \mathrm{EIG}_{1}, \mathrm{ATG}_{1}, \mathrm{GO}_{1}, \mathrm{GO}_{2}$ and $\mathrm{GO}_{3}$. which reveal the oxidation of graphite at this stage. The patterns of GOs show the presence of peaks regarding OFGs which reveals that disruption of graphitic stacking order with $\mathrm{sp}^{2}$ domains is increased by more oxidation and $\mathrm{sp}^{3}$ domains are replaced, instead. Different groups are observed in GO specimens: broad peak at $3600 \mathrm{~cm}^{-1}$ due to the $\mathrm{O}-\mathrm{H}$ bonding from hydroxyl groups; $\mathrm{C}=\mathrm{O}$ bonds from carbonyl or carboxyl groups $(\mathrm{O}=\mathrm{C}-\mathrm{OH})\left(1734 \mathrm{~cm}^{-1}\right), \mathrm{C}=\mathrm{C}$ bonds from unoxidized $\mathrm{sp}^{2}$ graphite domains $\left(1627 \mathrm{~cm}^{-1}\right), \mathrm{C}-\mathrm{O}-\mathrm{C}$ bonds from epoxides and $\mathrm{C}-\mathrm{O}$ bonds from carbonyls in the range below $1200 \mathrm{~cm}^{-1}$. The results of FTIR analysis confirm the presence of OFGs of hydroxyl, epoxy and carboxyl groups. Epoxy groups are more stable than hydroxyl or carboxyl groups because of two $\mathrm{C}-\mathrm{O}$ binding and a closed ring structure which could not be ruptured simply. The required energy of two $\mathrm{C}-\mathrm{O}$ bindings in epoxy is much more than what is required for one binding of oxygen and hydrogen in addition to one binding of oxygen and carbon in hydroxyl groups. Therefore, the content of hydroxyl groups is more than epoxy in GO structure.

The broad band for hydroxyl groups at $3600 \mathrm{~cm}^{-1}$ was attributed to three different $\mathrm{O}-\mathrm{H}$ bindings from alcohols and phenols attached to GO sheets and water trapped molecules between GO layers. Unfortunately, the presence of trapped water molecules was not shown in some structural models of GO, because the previous models only showed the structure of one GO sheet. The water molecules interact with oxygen or hydrogen atoms of OFGs with hydrogen bonding (figure 6). Therefore, these groups should be close together. The same idea was proposed by He et $a l^{12}$ and Lerf et $a l .{ }^{14}$ However, interactions between atoms were different in their work. They suggested bonding between carbon and hydrogen atoms in interlayer distance. These hydrogen bonds of functional groups and trapped water molecules between the layers define the interactions among layers of carbon planes. Between GO sheets, there is not only $\pi-\pi$ interactions as in graphite, but also strong hydrogen bonding. ${ }^{34}$ Therefore, it is better to show GO structure with two or more GO sheets to show the hydrogen bonding of functional groups and the presence of trapped molecules between GO layers.

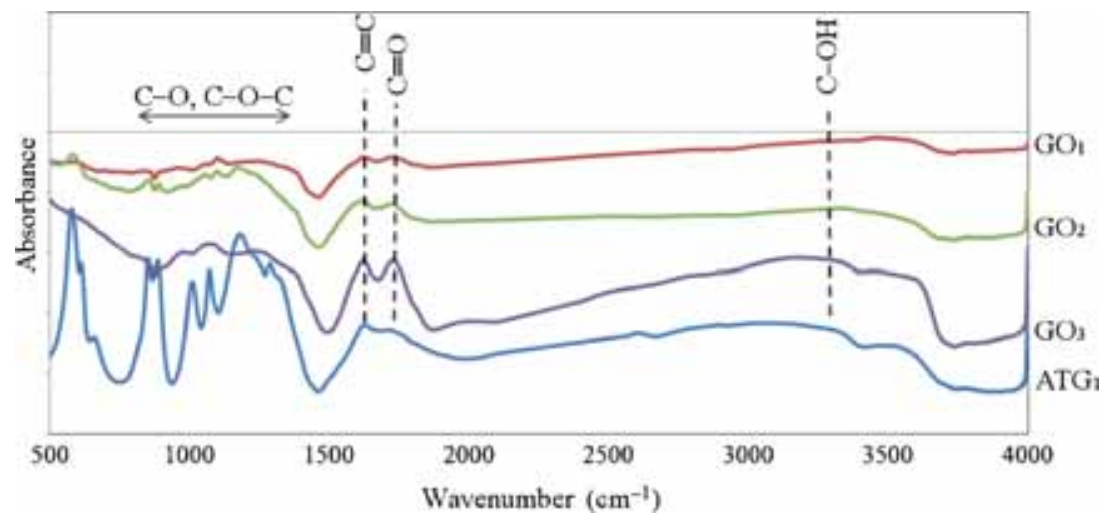

Figure 5. FTIR spectra of $\mathrm{ATG}_{1}, \mathrm{GO}_{1}, \mathrm{GO}_{2}$ and $\mathrm{GO}_{3}$. 
The content of OFGs in GO was also analysed using Boehm titrations. Total number of OFGs was $6.4 \mathrm{meq}^{-1}$. This value was less than what was measured for OFGs in GO. ${ }^{35}$ This is due to the lower degree of non-stoichiometric oxygen in larger GO sheets (average of $70 \mu \mathrm{m}$ in this work compared with $800 \mathrm{~nm}$ reported by Whitby et al. ${ }^{29,35-38}$ The content of carboxyl groups $\left(4.7 \mathrm{meq}^{-1}\right)$ was higher than phenols (1.5 meq $\left.\mathrm{g}^{-1}\right)$ and also, lactones $\left(0.2 \mathrm{meq} \mathrm{g}^{-1}\right)$. Therefore, carboxyl groups played a determinant role in $\mathrm{GO}$ structure.

For production of carboxyl groups, one carbon atom should be drawn from hexagonal carbon lattice with breaking of carbon $\mathrm{sp}^{2}$ bindings on the edges or one carbon should be drawn from inside of carbon lattice by breaking $\mathrm{C}-\mathrm{C}$ bonds which leaves behind a defect in the structure. Defects may also form during aggressive oxidation and sheet exfoliation by releasing $\mathrm{CO}$ and $\mathrm{CO}_{2} \cdot{ }^{39}$

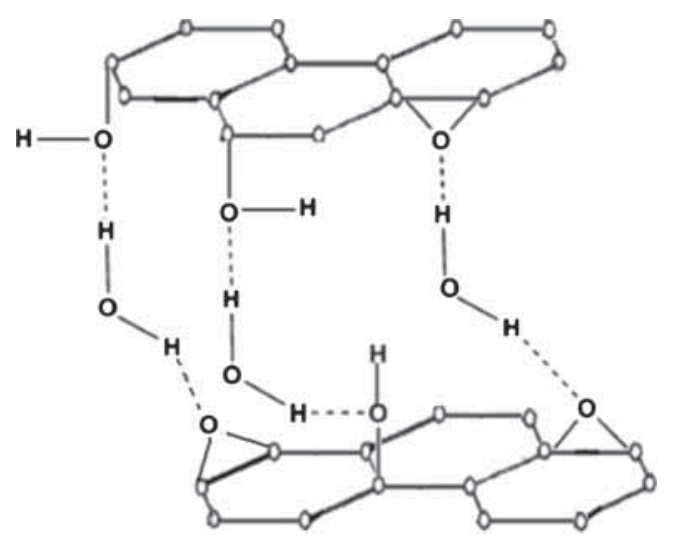

Figure 6. Schematic representation of interaction of water molecules with different oxygen functional groups in the structure of GO.
Ketones could not be determined using titration. Furthermore, it is not possible to distinct any obvious peak regarding ketones in FTIR graphs, as it is for the lactones, even though the content of lactones is measured by titration $(0.2$ meq $\mathrm{g}^{-1}$ ). Actually, carbonyl signals presented in FTIR studies are attributed to different functionalities including carboxyl groups, ketones and lactones. Ketones could only be present at the edges or at defect sites of $\mathrm{C}-\mathrm{C}$ bonds. ${ }^{17} \mathrm{It}$ means that due to the double bonding of a ketone group, carbons with just two bonds are hosted. Therefore, the chance for the presence of ketones is so little; especially in ultra-large GOs that the number of carbons at edges are much less than the inside area. It is the same for lactone rings.

Although the content of different functional groups could be estimated using experimental analysis of the structure of $\mathrm{GO}$, their real location on the carbonic plane is unknown. Configuration of OFGs inside carbon plane was suggested using energy calculation of structure with different configurations of epoxy and hydroxyl groups. ${ }^{18,40}$ Accordingly, OFGs tend to aggregate in carbon lattice and also, same close groups tend to place in opposite sites. Moreover, carboxyl groups and phenols prefer corner sites. ${ }^{41}$

Analysing the morphologies of GO in figure $2 \mathrm{~d}$ shows that some regions of GO layers are folded. It was mentioned previously $^{14}$ that the presence of $\mathrm{O}-\mathrm{H}$ bonding in carbon lattice results in some distortion of the structure near this bond.

According to above discussion, a modified structural model was proposed for GO, based on the model of LerfKlinowski $^{14}$ (figure 7 ). However, the proposed model has some differences with the model by Lerf-Klinowski. Lactones in addition to carboxyl groups, phenols and epoxides were presented in GO structure. OFGs were aggregated in some regions. The content of carboxyl groups was much higher than phenols and other groups. Defects were placed
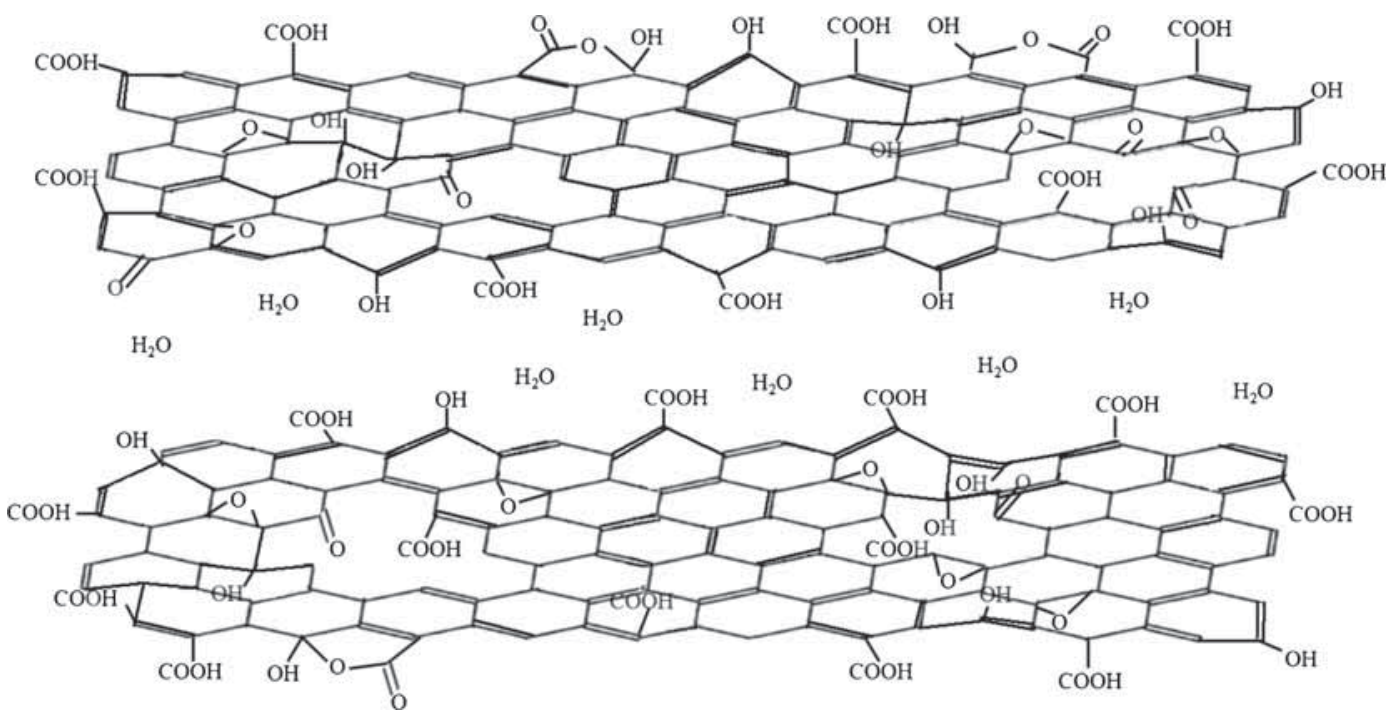

Figure 7. Proposed modified structure of graphene oxide. 
at different sites in hexagonal carbon lattice. The presence of trapped water molecules was shown between GO sheets which strongly affected the distribution of OFGs and their aggregation by hydrogen bonding. Moreover, compared to other models proposed for GO, based on the model by LerfKlinowski, Gao et a ${ }^{27}$ proposed GO structure with random distribution of OFGs. They did not place carboxyl groups and defects in their model.

It should be noted that the proposed structure does not have necessarily long-range order. Furthermore, it is not possible to determine the exact structure of GO due to the existence of complicated interactions among different atoms and groups. The proposed structure was just an attempt for proposing the structure of $\mathrm{GO}$ according to the experimental results and theoretical studies.

\section{Conclusions}

A modified structural model was proposed based on the model of Lerf-Klinowski. In this modified model, carboxyl groups, phenols, epoxides, lactones and ketones were found as functional groups in distorted hexagonal lattice in the place of hydroxyl groups. The content of carboxyl groups was much higher than phenols and other groups. Defects were placed in different sites in hexagonal carbon lattice. Trapped water molecules were placed between GO sheets which interacted with functional groups. The OFGs were placed close together due to strong hydrogen bonding between oxygen of one group with hydrogen from adjacent group and actually, agglomeration of OFGs happened at different regions on the GO planes.

\section{References}

1. Ding Y-H, Ren H-M, Chang F-H, Zhang P and Jiang Y 2013 Bull. Mater. Sci. 361073

2. Venugopal G, Krishnamoorthy K, Mohan R and Kim S-J 2012 Mater. Chem. Phys. 13229

3. Kang S-H, Fang T-H and Hong Z-H 2013 J. Phys. Chem. Solids 741783

4. Zhao N, Cheng X-N, Yang J, Yang M-X, Zheng S-H and Zhou Y-Z 2014 J. Phys. Chem. Solids 751141

5. Kong H X 2013 Curr. Opin. Solid State Mater. 1731

6. Barzegar F, Bello A, Fabiane M et al 2015 J. Phys. Chem. Solids 77139

7. Hofmann U and Holst R 1939 Ber. Dtsch. Chem. Ges. B 72754

8. Ruess G 1946 Monatsh. Chem. 76381

9. Nakajima T, Mabuchi A and Hagiwara R 1988 Carbon 26357

10. Hontoria-Lucas C, López-Peinado A J, López-González Jd D, Rojas-Cervantes M L and Martín-Aranda R M 1995 Carbon 331585
11. Mermoux M, Chabre Y and Rousseau A 1991 Carbon 29 469

12. He H, Riedl T, Lerf A and Klinowski J 1996 J. Phys. Chem. 10019954

13. Lerf A, He H, Riedl T, Forster M and Klinowski J 1997 Solid State Ion. 101-103 857

14. Lerf A, He H, Forster M and Klinowski J 1998 J. Phys. Chem. B 1024477

15. He H, Klinowski J, Forster M and Lerf A 1998 Chem. Phys. Lett. 28753

16. Lee D W, De Los Santos V L, Seo J W et al 2010 J. Phys. Chem. B 1145723

17. Szabó T, Berkesi O, Forgó P et al 2006 Chem. Mater. 182740

18. Yan J-A and Chou M Y 2010 Phys. Rev. B 82125403

19. Botas C, Álvarez P, Blanco C et al 2012 Carbon 50275

20. Lahaye R J W E, Jeong H K, Park C Y and Lee Y H 2009 Phys. Rev. B 79125435

21. Ijäs M and Harju A 2010 Phys. Rev. B 82235111

22. Wang L, Sun Y Y, Lee K et al 2010 Phys. Rev. B 82 161406R

23. Liu L, Wang L, Gao J, Zhao J, Gao X and Chen Z 2012 Carbon 501690

24. Kumar P V, Bardhan N M, Tongay S, Wu J, Belcher A M and Grossman J C 2014 Nat. Chem. 6151

25. Krishnamoorthy K, Veerapandian M, Yun K and Kim S J 2013 Carbon $\mathbf{5 3} 38$

26. Nakajima T and Matsuo Y 1994 Carbon 32469

27. Gao W, Alemany L B, Ci L and Ajayan P M 2009 Nat. Chem. 1403

28. Aboutalebi S H, Chidembo A T, Salari M et al 2011 Energy Environ. Sci. 41855

29. Aboutalebi S H, Gudarzi M M, Zheng Q B and Kim J-K 2011 Adv. Funct. Mater. 212978

30. Aboutalebi S H, Jalili R, Esrafilzadeh D et al 2014 ACS Nano 82456

31. Zhang T-Y and Zhang D 2011 Bull. Mater. Sci. 3425

32. Boehm H P 1994 Carbon 32759

33. Koch K R 1982 J. Chem. Educ. 59973

34. Acik M, Mattevi C, Gong C et al 2010 ACS Nano 45861

35. Whitby R L D, Korobeinyk A and Glevatska K V 2011 Carbon 49722

36. Jalili R, Aboutalebi S H, Esrafilzadeh D et al 2013 ACS Nano 73981

37. Chidembo A T, Aboutalebi S H, Konstantinov K, Wexler D, Liu H K and Dou S X 2014 Part. Part. Syst. Charact. 31 465

38. Jalili R, Aboutalebi S H, Esrafilzadeh D et al 2014 Mater. Horiz. 187

39. Erickson K, Erni R, Lee Z, Alem N, Gannett W and Zettl A 2010 Adv. Mater. 224467

40. Zhou S and Bongiorno A 2013 Sci. Rep. 32484

41. Lone B, Scheiner S and Kar T 2014 Carbon 80405 